\title{
Using a chronic hepatitis B Registry to support population-level liver cancer prevention in Sydney, Australia
}

This article was published in the following Dove Press journal: Clinical Epidemiology

\author{
Monica C Robotin, ${ }^{1-3}$ Ximena \\ Masgoret,' Mamta Porwal,4 \\ David Goldsbury, ${ }^{5}$ Chee \\ Khoo, ${ }^{6,7}$ Jacob George, ${ }^{2,3}$ \\ 'School of Medicine, The University of \\ Notre Dame Australia, Darlinghurst, \\ ${ }^{2}$ Faculty of Medicine, University of \\ Sydney, Camperdown, ${ }^{3}$ Storr Liver \\ Center, Westmead Institute for \\ Medical Research, Westmead Hospital, \\ Westmead, ${ }^{4}$ Australian School of \\ Graduate Management, University of \\ New South Wales, Kensington, ${ }^{5}$ Cancer \\ Council NSW, Woolloomooloo, ${ }^{6}$ Royal \\ Australasian College of General \\ Practitioners, Sydney, ${ }^{7}$ University of \\ Western Sydney, Macarthur, NSW, \\ Australia
}

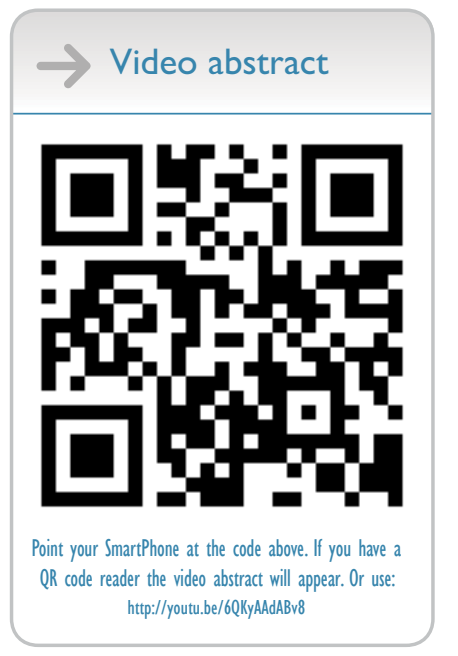

Correspondence: Monica C Robotin School of Medicine, The University of Notre Dame Australia, 160 Oxford Street, Darlinghurst, Sydney, NSW 2010, Australia Tel +6I 282044462

Fax +6I 293577680

Email monica.robotin@nd.edu.au
Background: Approximately 1\% of Australians have chronic hepatitis B (CHB), which disproportionately affects people born in hepatitis B-endemic countries. Currently, approximately half of the people affected remain undiagnosed and antiviral treatment uptake is suboptimal ( $\sim 5 \%)$. This increases the likelihood of developing end-stage disease complications, particularly hepatocellular cancer (HCC), and largely accounts for the significant increases in HCC incidence and mortality in Australia over the last decades. As our previous economic modeling suggested that CHB screening and treatment is cost-effective, we tested the feasibility of a primary carebased model of CHB diagnosis and management to prevent HCC.

Materials and methods: From 2009 to 2016, the B Positive program trialed a CHB screening and management program in an area of high disease prevalence in Sydney, Australia. Trained local primary care providers (general practitioners) screened and managed their CHB patients using a purpose-built CHB Registry and a risk stratification algorithm, which allocated patients to ongoing primary care-based management or specialist referral.

Results: The program enrolled and followed up $>1,500$ people ( $25 \%$ of the target population). Their median age was 48 years, with most participants being born in China (50\%) or Vietnam (32\%). The risk stratification algorithm allocated most Registry participants ( $n=847$ or $79 \%)$ to primary care-based management, reducing unnecessary specialist referrals. The level of antiviral treatment uptake in Registry patients was $18 \%$, which was the optimal level in this population group.

Conclusion: This pilot program demonstrated that primary care-based hepatitis B diagnosis and management is acceptable to patients and their care providers and significantly increases compliance with treatment guidelines. This would suggest that scaling up access to hepatitis B treatment is achievable and can provide a means to operationalize a population-level approach to CHB management and liver cancer prevention.

Keywords: hepatitis B Registry, primary care, cancer prevention, antiviral treatment, risk stratification

\section{Introduction}

The last four decades have witnessed an unprecedented increase in liver cancer incidence in Australia, elevating a relatively rare cancer to one of the top 10 causes of cancer death. ${ }^{1}$ Hepatocellular cancer or HCC (by far the most common form of liver cancer) is frequently diagnosed late and given its poor response to therapy, incidence of $\mathrm{HCC}$ almost matches disease mortality, with a median survival of just 5.1 months. ${ }^{2}$

Unlike many cancers, $\mathrm{HCC}$ is largely preventable, as $\sim 80 \%$ cases are attributable to chronic hepatitis $\mathrm{B}(\mathrm{CHB})$ or $\mathrm{C}$ infection, ${ }^{3}$ both of which can be prevented and/or 
successfully mitigated. The Global Burden of Disease Study found that approximately half of the global liver cancer mortality is attributable to hepatitis $\mathrm{B}$ infection, with smaller fractions due to hepatitis $\mathrm{C}$ and alcohol. ${ }^{4}$ In the absence of vaccination, early disease acquisition is common in endemic areas and despite remaining asymptomatic for decades, $\mathrm{CHB}$ eventually leads to cirrhosis or hepatitis B-related liver in $\sim 25 \%$ of cases. $^{5}$ Approximately 800,000 deaths/year are attributable to the infection and its complications globally, ${ }^{4,5}$ as chronically infected individuals have a 100-fold higher HCC risk than the people not infected. ${ }^{6}$

As hepatitis B infection is endemic in many South East Asian countries, some Pacific Islands and some African countries, increased migration has been associated with rising $\mathrm{HCC}$ rates in countries with large migrant populations in North America, Western Europe and Australia. ${ }^{7-9}$ In Australia's most populous State (New South Wales [NSW]), 47\% of liver cancer diagnoses occur in migrants, with people born in a hepatitis B-endemic country being 6-13 times more likely to receive an HCC diagnosis than other (nonindigenous) Australians. ${ }^{8}$

It is estimated that of the $\sim 218,000$ Australians with $\mathrm{CHB}$, $56 \%$ were born overseas. ${ }^{10}$ Similar to US data, ${ }^{11}$ over $95 \%$ of chronic infections adding to the general pool in Australia annually result from migration from endemic countries, rather than "domestic infections" failing to clear, ${ }^{10}$ suggesting that the highest yield in identifying people with $\mathrm{CHB}$ infection would result from systematically screening people born in hyperendemic countries. ${ }^{12}$ While all people with $\mathrm{CHB}$ require lifelong monitoring, only $15 \%-25 \%$ of people chronically infected require antiviral treatment. ${ }^{13}$

Hepatitis B is a notifiable disease in Australia, and medical care and antiviral treatment are available through the national health care system (Medicare), but significant gaps remain in its diagnosis and treatment. In 2011, it was estimated that just over half (57\%) of Australians with CHB $(\sim 125,000$ people) had been diagnosed and only $8 \%$ of them were receiving annual viral load testing and used to monitor the disease stage and need for antiviral treatment. ${ }^{14}$ In 2013 , it was estimated that overall antiviral treatment uptake was $\sim 5 \% .^{15}$ Migrants are more likely to experience barriers to being diagnosed and treated for hepatitis B at provider, patient and health system levels. ${ }^{16}$ Disease awareness and knowledge remain low in high-risk populations and among their care providers, ${ }^{17}$ and consequently, $\mathrm{CHB}$ testing, management and linkage to care remain unsystematic. However, as $\mathrm{CHB}$ is a chronic disease, requiring regular follow-up, general practitioners (GPs) could play a pivotal role in disease management and reduce the demands on specialist clinics.
To ascertain whether a population-level approach to $\mathrm{CHB}$ management and HCC prevention is feasible in an Australian context, we conducted economic modeling to ascertain the costs, benefits and workforce implications of different CHB management strategies. We found that a primary carebased strategy, where GPs screen, manage and/or refer CHB patients according to their level of HCC risk was the most cost-effective management option. ${ }^{18}$ This strategy, which we termed an HCC prevention strategy, could reduce cases of cirrhosis by $52 \%$, HCC diagnoses by $47 \%$ and CHB-related deaths by $56 \%$, compared to the current practice, of unsystematic CHB screening and treatment. ${ }^{18}$

We modeled the potential impact of this model of care in South Western Sydney, a region with the largest number of $\mathrm{CHB}$ diagnoses ${ }^{15}$ and highest $\mathrm{HCC}$ incidence in NSW. ${ }^{19}$ In the Fairfield Local Government Area, overall CHB prevalence is estimated at $2.7 \%$, and $76.5 \%$ of those affected were born overseas, mostly in Vietnam and China. ${ }^{15}$ Our economic model estimated that of local residents with $\mathrm{CHB}, \sim 5,800$ were born in China or Vietnam and were aged $>35$ years, ${ }^{20}$ when the likelihood of developing disease complications increases significantly. ${ }^{21}$ Using Australian CHB management guidelines, our economic model predicted that $19 \%$ of this patient group were candidates for antiviral treatment. ${ }^{22}$

We subsequently piloted a population-based intervention (called B Positive) in the Fairfield Local Government Area, seeking to increase $\mathrm{CHB}$ awareness in high-risk populations and to educate and support local GPs to become proficient in CHB testing and management. ${ }^{23}$ We developed and distributed educational resources in English, Vietnamese, Cantonese and Mandarin and invited local GPs to participate in hepatitis B-themed Continuing Medical Education (CME) events aimed at understanding their training needs and supporting them to incorporate hepatitis B practice guidelines into their practice. ${ }^{24}$ We also developed a customized disease Registry, which provided logistical GP support to enroll and follow-up their CHB patients.

This paper aims to characterize the Registry participants' demographic and disease characteristics and to compare the actual management pathways chosen by participating GPs with the B Positive program algorithm.

\section{Materials and methods \\ Participant selection}

All GPs practicing in the program area were invited to attend a series of free CME seminars on hepatitis B topics and participants were provided with a copy of a monograph on hepatitis B, written especially for primary care providers. ${ }^{25}$ 
Over the first 12 months of the pilot, a total of 70 local GPs (representing a fifth of GPs registered with the relevant Division of General Practice) attended the CME sessions. ${ }^{26}$ Most participants were male, spoke more than one language with their patients, self-identified as Asian Australians and had graduated 20 or more years before. ${ }^{24}$ The majority of GPs enrolling patients in the Registry were recruited through this avenue and by detailing of GPs with similar ethnic affiliations. Participating GPs informed their eligible patients about the option of enrolling in the CHB Registry and documented verbal informed consent in their medical records. GPs identified potential participants through medical chart review (for patients already diagnosed with $\mathrm{CHB}$ ) or targeted screening of high-risk patient groups, according to local clinical practice guidelines. ${ }^{27}$ Enrollment was offered to all patients with a confirmed CHB diagnosis, with case finding focused on individuals aged $\geq 35$ years and born in hepatitis B-endemic countries. Exclusion criteria included pregnant or breastfeeding women and individuals unable to provide informed consent.

\section{Participant follow-up}

GPs completed clinical record forms and sent these to Registry staff (consisting of the program manager, a clinical nurse consultant and a database administrator), who checked data integrity, entered it into the database for analysis and sent patient reminders to attend biannual GP follow-up visits. Prior to each GP visit, participants received a Registry-generated reminder letter and a referral to a local pathology collection service.

\section{Clinical assessment}

The GP conducted a clinical assessment and reviewed investigation results, including hepatitis B virus (HBV) serology, alanine aminotransferase (ALT) and serum alphafetoprotein (AFP) levels, and liver ultrasound (US) reports. HBV DNA (or viral load [VL]) levels were monitored yearly in treatment-naïve participants and as indicated for those on treatment. Follow-up plans (ongoing GP follow-up or specialist referral) were documented at each visit. Additional data collected included general demographic information (name, contact details, date and country of birth, ethnic origin and year of arrival in Australia), limited risk factor information (family history of HCC and presence of diabetes mellitus, overweight/obesity and dyslipidemia) and documentation of antiviral treatment.

Decisions regarding the type of follow-up were informed by the B Positive CHB management algorithm (Figure 1), which uses VL and ALT levels to stratify patients into three management pathways. Participants with VL $<2,000 \mathrm{IU} /$ $\mathrm{mL}$ and "normal" ALT levels (defined as $<1.5 \times$ the upper limit of the normal [ULN] range) receive routine hepatitis care from their GP, which involves 6-monthly GP visits and blood tests (ALT; hepatitis B virus surface antigen [HBsAg], hepatitis B e antigen [HBeAg], anti-HBe and AFP levels) and annual HBV DNA checks and liver US (if aged $>40$ years). Participants with elevated VL $(\geq 2,000 \mathrm{IU} / \mathrm{mL})$ and elevated ALT levels ( $>1.5 \times$ ULN) are referred for specialist assessment, while those with "normal" ALT levels and VL $\geq 2,000$ $\mathrm{IU} / \mathrm{mL}$ remain under GP follow-up and receive enhanced surveillance, which follows the same protocol as routine hepatitis care, as well as biannual liver US to rule out HCC. ${ }^{18}$ To simplify communication around disease monitoring, the Registry assigns a color code to each management pathway. The yellow pathway is applicable to people receiving routine hepatitis care (i.e., with "normal" ALT and low or undetectable HBV DNA levels). The orange pathway (patients with elevated VL and "normal" ALT levels) relates to GP-led enhanced HCC surveillance. Patients with elevated ALT levels require specialist referral through the red (high ALT and VL levels) or purple (high ALT and VL $<2,000 \mathrm{IU} / \mathrm{mL}$ ) pathway (Figure 1). We compared the predicted management pathways to GPs actual decisions, enabling us to conduct a clinical audit of CHB management patterns in these practices.

The B Positive program was approved by the Human Research Ethics Committee of the South-West Sydney Area Health Service. It recruited participants from November 2011 to July 2016. This pilot program was funded by a not-forprofit organization (Cancer Council NSW) to demonstrate program feasibility and acceptability, on an understanding that continued funding would be provided by health authorities.

\section{Data analysis}

Data were entered into a FileMaker Pro v11 database, deduplicated and cleaned, and missing data fields verified against the medical records of the referring GP. Data were extracted and analyzed using the SPSS 15.0 statistical software. Continuous variables were characterized using median and interquartile range (IQR) and compared using the MannWhitney $U$ test. Chi-square tests were used for comparing categorical variables. Associations between age, gender and VL were analyzed and ALT and HBV DNA levels tabulated using the cut-offs recommended by Australian clinical practice guidelines. ${ }^{27}$ Registry staff reviewed the blood tests and documented GP management decisions and compared them to those recommended by the B Positive algorithm. 


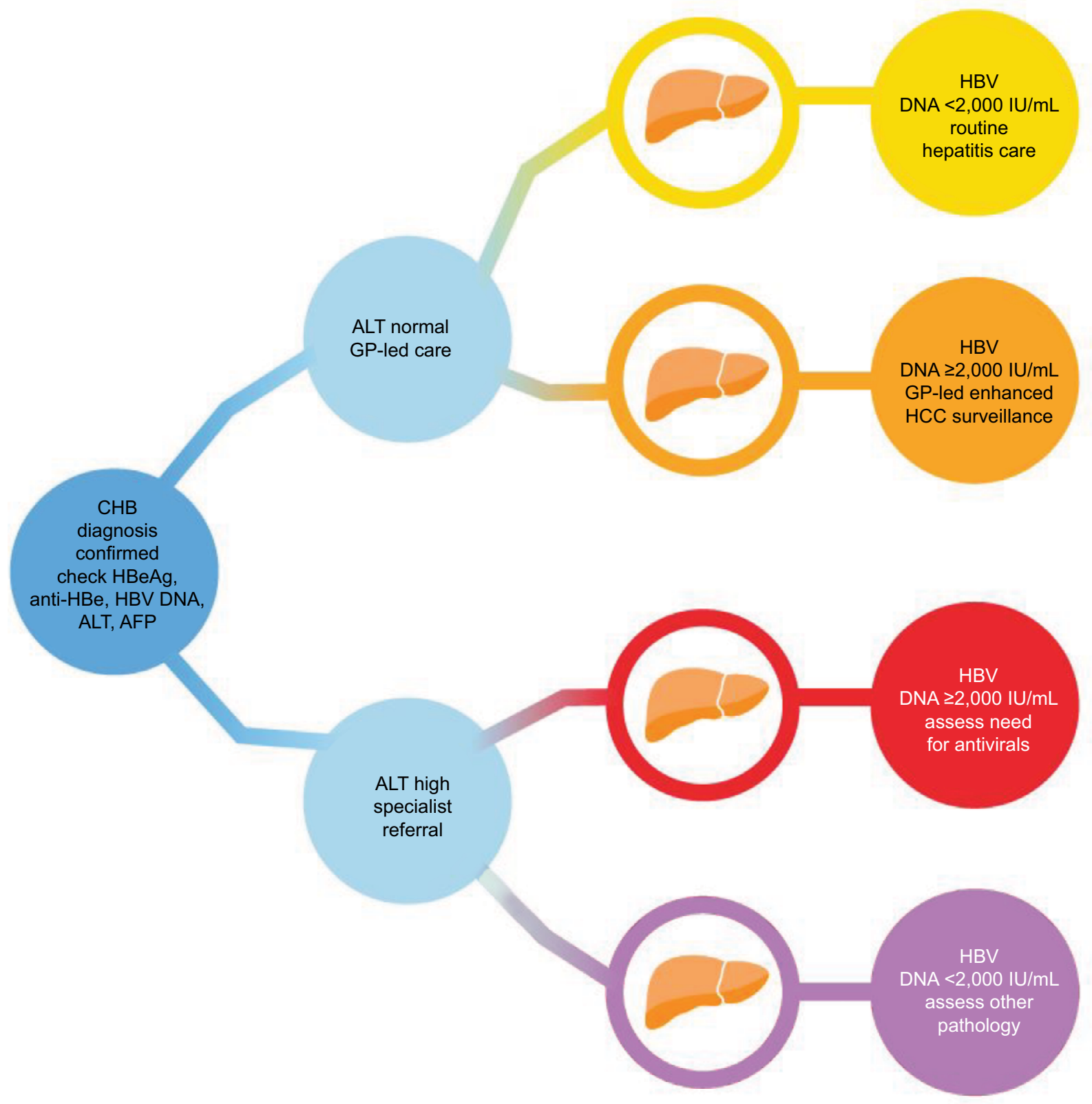

Figure I The chronic hepatitis B management protocol used by the B Positive disease Registry.

Notes: GP-led care: routine care: primary care-based 6-monthly ALT and annual HBV DNA, HBsAg, HBeAg and anti-HBe; 6-monthly AFP and annual liver US (if aged >40 years); GP-led HCC surveillance: same protocol as routine care, but 6-monthly liver US (if aged $>40$ ). Specialist referral: referral for further investigations and management. ALT high: $\geq 1.5 x$ upper limit of normal range.

Abbreviations: AFP, alpha-fetoprotein; ALT, alanine aminotransferase; anti-HBe, antibodies to hepatitis B envelope antigen; CHB, chronic hepatitis B; GP, general practitioner; $\mathrm{HBeAg}$, hepatitis B e antigen; HBV DNA, hepatitis B virus DNA level; HCC, hepatocellular carcinoma; US, ultrasound.

\section{Results}

By January 2016, 1,500 people (25\% of the target population) were enrolled in the Registry by our 70 participating GP partners (20\% of local GPs). The number of patients enrolled per GP ranged from 1 to 185 (median 8 patients per GP).

\section{Participants' demographic characteristics}

The demographic and clinical characteristics of Registry participants at enrollment are summarized in Table 1. As the
Registry sought to enroll participants older than 35 years, $82 \%$ were in this age group. Median age at enrollment was 48 years (IQR 38-56), with no statistically significant difference between male (median 49 years, IQR 38-57) and female (median 47 years, IQR 37-56) participants (Mann-Whitney $Z=-1.1, p=0.25$ ).

Country of birth information was available for $96 \%$ of enrollees. The majority were born in mainland China (39\%) or Vietnam (32\%), with a further $11 \%$ born in Hong Kong 
Table I Demographic and clinical characteristics of the first I,500 patients enrolled in the CHB disease Registry

\begin{tabular}{|c|c|c|}
\hline Characteristic & Number & $\%$ \\
\hline \multicolumn{3}{|l|}{ Gender } \\
\hline Male & 693 & 46 \\
\hline Female & 807 & 54 \\
\hline \multicolumn{3}{|l|}{ Age groups (years) } \\
\hline$<35$ & 273 & 18 \\
\hline $35-50$ & 610 & 41 \\
\hline$>50$ & 616 & 41 \\
\hline Not recorded & 1 & 0 \\
\hline \multicolumn{3}{|l|}{ Country of birth } \\
\hline Mainland China & 592 & 39 \\
\hline Vietnam & 487 & 32 \\
\hline Hong Kong/Taiwan & 163 & II \\
\hline Other Asian countries & 120 & 8 \\
\hline Pacific Islands & 44 & 3 \\
\hline Australia & 10 & I \\
\hline Other & 24 & 2 \\
\hline Not recorded & 60 & 4 \\
\hline \multicolumn{3}{|l|}{$\mathrm{HBeAg}$ status } \\
\hline HBeAg negative & 1,283 & 86 \\
\hline $\mathrm{HBeAg}$ positive & 156 & 10 \\
\hline Not recorded & 61 & 4 \\
\hline \multicolumn{3}{|l|}{ DNA level (IU/mL) } \\
\hline Undetectable $(<20)$ & 397 & 26 \\
\hline $20-2,000$ & 586 & 39 \\
\hline $2,000-20,000$ & 183 & 12 \\
\hline$>20,000$ & 172 & 12 \\
\hline Not recorded & 162 & II \\
\hline \multicolumn{3}{|l|}{ ALT levels } \\
\hline$<1.5 \times$ above normal range ${ }^{a}$ & 1,152 & 77 \\
\hline I.5-2.0× above normal range & 175 & 12 \\
\hline$>2.0 \times$ above normal range & 155 & 10 \\
\hline Not recorded & 18 & I \\
\hline
\end{tabular}

Note: a Normal ALT levels defined as $<45 \mathrm{IU} / \mathrm{L}$ in males and $<30 \mathrm{IU} / \mathrm{L}$ in females. Abbreviations: ALT, alanine aminotransferase; $\mathrm{CHB}$, chronic hepatitis $\mathrm{B} ; \mathrm{HBeAg}$, hepatitis B envelope antigen.

or Taiwan. A small proportion originated from other Asian countries (Cambodia, Philippines, Korea, Laos, Malaysia and Burma), the Pacific Islands or Australia. Duration of residence in Australia was available for $75 \%$ of participants born in China or Vietnam; people born in Vietnam had lived in Australia for a significantly longer period (median 23 years, IQR 14-29) than those born in China (median 16 years, IQR 9-23; Mann-Whitney $Z=-7.18, p \leq 0.001)$.

\section{CHB disease characteristics}

Most participants $(1,283$ or $86 \%)$ were seronegative for $\mathrm{HBeAg}$, a finding consistent with previous studies. ${ }^{21}$ The proportion of $\mathrm{HBeAg}$-seronegative patients increased with age, ranging from $14 \%$ for those aged $<35$ years to $35 \%$ for those aged $35-50$ years and $37 \%$ for those $>50$ years of age. Two thirds had HBV DNA levels in the undetectable or $<2,000 \mathrm{IU} / \mathrm{L}$ range, and nearly a quarter had VL levels $\geq 2,000 \mathrm{IU} / \mathrm{mL}$ (Table 1 ). ALT levels ranged from 2 to 333 $\mathrm{IU} / \mathrm{mL}$ (median 24). ALT levels were within $1.5 \times$ of the normal range for approximately three-quarters of the participants. Enrollment AFP levels were documented in $82 \%$ of patients and ranged from 0.5 to $252 \mathrm{ng} / \mathrm{L}$, with a median of $2 \mathrm{ng} / \mathrm{L}(\mathrm{IQR}=1)$. Three patients were diagnosed with HCC at enrollment.

A family history of HCC was documented in $4 \%$ of participants, while $7 \%$ had diabetes mellitus and $16 \%$ had dyslipidemia. As liver US reports were only available for 33\% of the participants, the results of US examinations were not factored into this analysis.

A total of 1,230 participants $(82 \%)$ were not receiving antiviral treatment at enrollment, and complete information to allow HCC risk stratification using the B Positive algorithm was available for 1,077 of the antiviral-naïve patients (72\%). Based upon the B Positive algorithm, we ascertained that $79 \%$ of the participants $(n=847)$ could remain under GP care, with the majority (58\%) suitable for routine surveillance (yellow cells in Table 2) and 21\% requiring enhanced CHB surveillance (orange cells in Table 2). Relatively few people in this community-based sample had elevated ALT levels $(21 \%)$, where clinical practice guidelines recommend specialist referral (red and purple cells in Table 2).

While people with normal ALT levels can remain under GP care according to the B Positive care model, the data collected indicated that $19 \%$ of the enrollees with normal ALT levels and HBV DNA levels $<2,000 \mathrm{IU} / \mathrm{mL}$ (117 patients) and $40 \%$ of those with HBV DNA levels $>2,000 \mathrm{IU} / \mathrm{mL}$ (87 patients) were referred to specialists. Conversely, although specialist assessment is recommended for patients with ALT levels $>1.5 \times$ ULN, of the 230 participants in this category, only $58 \%$ of those with increases in both ALT and VL were referred, as were $18 \%$ of those with high ALT and undetectable or low DNA levels (Table 2).

Of the 270 participants receiving antiviral therapy (18\% of the entire cohort), complete documentation of the test results at enrollment was available in 254 (94\%). Over two thirds $(68 \%)$ had an undetectable VL, 14\% had low level of VL $(<2,000 \mathrm{IU} / \mathrm{mL})$ and $12 \%$ had VL exceeding 2,000 IU/mL. Most people on treatment who had undetectable VL (168 participants or $66 \%$ ) also had normal ALT levels.

\section{Discussion}

The B Positive program demonstrated that a primary carebased model of CHB detection and management is feasible and acceptable to local primary care providers and their 
Table 2 Number and proportion of patients managed by GPs, or referred to specialists in the B Positive Registry, based upon ALT levels and $\mathrm{VL}^{\mathrm{a}}$

\begin{tabular}{|c|c|c|c|c|c|c|c|}
\hline \multirow{2}{*}{$\begin{array}{l}\text { ALT } \\
\text { level }\end{array}$} & \multicolumn{4}{|l|}{ VL level } & \multirow{2}{*}{$\frac{\text { Total }}{\text { n (\%) }}$} & \multicolumn{2}{|c|}{ Management } \\
\hline & $\begin{array}{l}\text { Not } \\
\text { detectable } \\
\text { n (\%) }\end{array}$ & $\begin{array}{l}<2,000 \\
\mathrm{IU} / \mathrm{mL} \\
\mathrm{n}(\%)\end{array}$ & $\begin{array}{l}2,000-20,000 \\
I U / m L \\
n(\%)\end{array}$ & $\begin{array}{l}>20,000 \\
\mathrm{IU} / \mathrm{mL} \\
\mathrm{n}(\%)\end{array}$ & & $\begin{array}{l}\text { Algorithm- } \\
\text { based care } \\
\text { provider }\end{array}$ & $\begin{array}{l}\text { Observed number } \\
\text { of specialist referrals, } \\
\text { n (\%) by category }\end{array}$ \\
\hline \multirow[t]{2}{*}{$<1.5 \times \mathrm{N}$} & $185(17)$ & $443(4 I)$ & $137(13)$ & $82(8)$ & $847(79)$ & GP & 117 (19) \\
\hline & & & & & & & $87(40)$ \\
\hline \multirow[t]{2}{*}{$\geq 1.5 \times \mathrm{N}$} & $28(3)$ & $102(9)$ & $33(3)$ & $67(6)$ & $230(21)$ & Specialist & $58(58)$ \\
\hline & & & & & & & $24(18)$ \\
\hline Total & 213 & 545 & 170 & 149 & $\mathrm{I}, 077$ & & 286 \\
\hline
\end{tabular}

Notes: Yellow cells: routine GP surveillance; orange cells: enhanced GP surveillance; red and purple cells: specialist assessment. Normal ALT levels defined as <45 IU/L in males and $<30 \mathrm{IU} / \mathrm{L}$ in females. analysis restricted to the $\mathrm{I}, 077$ patients not receiving antiviral therapy and with complete data. Columns $2-5$ categorize patients using the $B$ Positive algorithm; the last column lists patients in each category referred to specialists

Abbreviations: ALT, alanine aminotransferase; GP, general practitioner; VL, viral load.

patients. The CHB Registry data we collected enabled an ascertainment of the different CHB disease categories in a population-based sample, which guides the level of care patients require and assists workforce planning as well as the monitoring of the uptake of guideline-based CHB management at a primary care level.

Despite a relatively short period of activity ( $<5$ years), the program reached $25 \%$ of its target population and achieved an antiviral therapy uptake of $18 \%$, which is over 3 -fold higher than the average antiviral uptake in Australia. ${ }^{19}$ Furthermore, the Registry data validated the role of economic modeling in informing policy. Our economic model had predicted that assuming a $25 \%$ program uptake, 271 patients (19\%) would require antiviral therapy, ${ }^{22} 2$ would be diagnosed with $\mathrm{HCC}$ at enrollment ${ }^{20}$ and $81 \%$ could continue to be cared for by their GP through routine hepatitis care $(50 \%)$ or enhanced HCC surveillance (31\%). ${ }^{22}$ These predictions proved extremely accurate: in this cohort, 270 patients $(18 \%)$ were receiving antiviral treatment and 3 patients were diagnosed with HCC at enrollment.

These results are even more remarkable given the fact that the project area has the lowest socioeconomic indicators in the Sydney region and $\sim 30 \%$ of the local population speaks little or no English, ${ }^{28}$ which further limits their opportunities to engage with the health system. ${ }^{15,29}$ This primary care-based model of CHB screening and treatment also capitalized on the language skills of local GPs, $91 \%$ of whom speak at least one additional language with their patients. ${ }^{24}$ Having trained GPs delivering language-concordant hepatitis care to their patients enhances continuity of care and avoids unnecessary specialist referrals, which are fraught with patient-doctor communication challenges, even when interpreters are available. ${ }^{30}$

A recent review of cancer screening interventions found that the most effective approaches to increase compliance with guidelines employed postal or telephone reminders and scheduled (rather than open) medical appointments. ${ }^{31}$ The B Positive Registry used scheduled reminders to ensure patients benefited from regular follow-ups and timely treatment initiation.

To the best of our knowledge, this level of CHB antiviral treatment uptake has not been reported by any other organized disease control program in Australia or elsewhere. Some community-based initiatives in the USA sought to offer a complete CHB care package, such as the BfreeNYC program in New York ${ }^{32}$ and the San Francisco Hepatitis B Free program. ${ }^{33}$ Despite reaching and screening large numbers of people, they experienced challenges in referral to care: $6.5 \%$ of people with CHB were referred by the San Francisco program ${ }^{34}$ and this figure was not reported by the New Yorkbased program. To improve disease surveillance, the city of San Francisco established a population-based CHB Registry, collecting risk factor and demographic information about persons with chronic viral hepatitis, to help characterize the local burden of CHB infection, but as far as we can ascertain, local treatment uptake was not recorded. ${ }^{35}$

Outside the REVEAL cohort in Taiwan, population-level data on CHB disease stage and HCC risk are limited and the B Positive Registry successfully addresses this information gap. In the REVEAL CHB study, $85 \%$ of participants were $\mathrm{HBeAg}$ negative and $73 \%$ had low to undetectable $\mathrm{VL} ;{ }^{36}$ the corresponding Australian rates are $86 \%$ and $62 \%$, respectively. Similar disease characteristics found in our multiethnic study could indicate that disease characteristics are similar in migrants originating from Asian countries and may justify careful extrapolation of data from Asian studies to Asian-born Australians.

Despite a relatively young median age (48 years) of patients in this cohort, a sizable proportion ( $7 \%$ ) had diabetes 
and 16\% had dyslipidemia. Therefore, including CHB into chronic disease management plans may offer additional benefits, as the management of metabolic comorbidities is anticipated to become a major challenge in reducing $\mathrm{HCC}$ burden in the future. ${ }^{37}$ The Bettering the Evaluation and Care of Health database, which monitors General Practice activity in Australia, found that $55 \%$ of General Practice consultations in 2014-2015 were related to the management of chronic conditions, particularly hypertension, depressive disorders, diabetes, arthritis and lipid disorders. ${ }^{38}$ Therefore, seeking to integrate $\mathrm{CHB}$ management with other chronic conditions makes good economic and clinical sense.

Our economic model predicted that local specialist services would experience a substantial increase in workload under the existing care model: at $25 \%$ of program uptake, local specialists would need to provide an additional 760 consultations annually for patients with CHB. ${ }^{20}$ Therefore, our GP collaborators can make a significant contribution toward reducing the burden on specialist services.

\section{Data limitations}

The project had to balance the needs for recording detailed clinical information with the willingness of participating GPs to take the additional time to document it. We suspect that some fields (i.e., a family history of HCC or of comorbidities) may have been underreported. Similarly, liver US results at enrollment were only documented in $33 \%$ of participants, so the presence of cirrhosis could not be reliably inferred from these data. As liver US is part of the management algorithm and is increasingly supplemented by liver elastography, these investigations are slowly becoming routine in evaluating patients with $\mathrm{CHB}$.

The proportion of tertiary referrals in this early sample was higher than anticipated, with $19 \%$ of patients fitting the criteria for routine surveillance and $40 \%$ of those in the enhanced surveillance group referred to specialist care. It is likely that some of the specialist referrals in the "yellow" or "orange" categories were prompted by the need for a Fibroscan examination (which was only available in the tertiary sector until recently). The limited data fields in the Registry preclude a clear understanding for the reason for referral (or nonreferral) of some of these patients. It may be possible that some of the "purple" patients had previously been referred to specialists for a pre-existing condition or to follow-up suspicious findings on an US examination. We anticipate that in time, increased familiarity with the triage system and increased access to community-based Fibroscan examinations will further reduce some of these referrals. The last few years have witnessed significant progress in hepatitis
B care, with GPs now able to prescribe antiviral treatments and care for their own patients, reducing the reliance on the tertiary sector for ongoing management of uncomplicated CHB cases.

Since the commencement of the B Positive program, more sophisticated predictive scoring systems for $\mathrm{HCC}$ and cirrhosis have become available. ${ }^{39-41}$ While most are derived from hospital-based cohorts, the Risk Estimation for HCC in Chronic Hepatitis B score was developed using communitybased data and included patients without cirrhosis. The REVEAL B system uses five clinical variables to calculate the risk and is applicable to a primary care setting. ${ }^{42}$ However, it is unlikely that the average Australian GP will use a risk calculator: Bettering the Evaluation and Care of Health data found that in 2014-2015, the mean length of a GP consultation was 14.4 minutes and most consultations required the management of more than one medical condition. ${ }^{38}$ To ensure continued GP engagement, simple and expeditious tools are needed to guide decision making, particularly for conditions they encounter infrequently, such as CHB. Even in a relatively high disease prevalence area, such as Fairfield, the median number of CHB cases per GP was just 8 . To ensure acceptability by time-poor GPs, the disease Registry collected a limited number of data fields, which, in conjunction with the color-coded decision support tool, enabled the GPs to conduct evidence-based HCC risk assessment and patient triage. While we cannot rule out the fact that some of these referrals were prompted by clinical information not captured by the Registry (and therefore were not "inappropriate referrals"), with increased familiarity with CHB management, we anticipate that specialist referrals for patients with inactive/ uncomplicated $\mathrm{CHB}$ will reduce further and referral of highrisk patients will increase.

\section{Conclusion}

The B Positive program demonstrated that a populationlevel approach to disease control is cost-effective, feasible and acceptable to patients and their health care providers. However, achieving population-level impact is contingent upon ongoing program funding and support and government endorsement. In its absence, results of pilot projects will not translate into the hoped-for reductions in liver cancer burden.

\section{Acknowledgments}

We thank all the Registry participants and their treating GPs for their participation and support of the B Positive program. We are grateful to the members of the project Steering Committee for advice on Registry set-up, data collection and study procedures and to Dr Andrew Penman, the former 
CEO of Cancer Council NSW, for his unstinting support of the program. JG is supported by the Robert W Storr Bequest to the Sydney Medical Foundation, University of Sydney; a National Health and Medical Research Council of Australia (NHMRC) Program Grant (1053206) and project grants (APP1107178 and APP1108422); and a Translational Cancer Research Center grant from the Cancer Institute NSW.

\section{Author contributions}

MCR conceived the study, contributed to the development of the economic model and data collection and analysis, and was responsible for drafting the manuscript. XM was primarily responsible for data collection and analysis and contributed to manuscript preparation. MP contributed to data collection and analysis, and manuscript preparation. DG assisted with data analysis and critically reviewed the manuscript. CK contributed to the design of the study, assisted with GP recruitment and support during the project and critically reviewed the manuscript. JG contributed to the design of the study and the economic model and was responsible for the overall supervision of the project. All authors contributed toward data analysis, drafting and revising the paper and agree to be accountable for all aspects of the work.

\section{Disclosure}

The authors report no conflicts of interest in this work.

\section{References}

1. AIHW (Australian Institute of Health and Welfare), AACR (Australasian Association of Cancer Registries). Cancer in Australia: an overview, 2012. Canberra: AIHW; 2012.

2. Gellert L, Jalaludin B, Levy M. Hepatocellular carcinoma in Sydney South West: late symptomatic presentation and poor outcome for most. Intern Med J. 2007;37(8):516-522.

3. Lavanchy D. Hepatitis B virus epidemiology, disease burden, treatment, and current and emerging prevention and control measures. $J$ Viral Hepat. 2004;11(2):97-107.

4. Lozano R, Naghavi M, Foreman K, et al. Global and regional mortality from 235 causes of death for 20 age groups in 1990 and 2010: a systematic analysis for the Global Burden of Disease Study 2010. Lancet. 2012;380(9859):2095-2128.

5. World Health Organization (WHO). Immunization surveillance, assessment and monitoring. Available from: http://www.who.int/immunization_monitoring/burden/field_studies/en/index.html. Accessed October 31, 2006.

6. Beasley RP. Hepatitis B virus. The major etiology of hepatocellular carcinoma. Cancer. 1988;61(10):1942-1956.

7. El-Serag HB. Epidemiology of viral hepatitis and hepatocellular carcinoma. Gastroenterology. 2012;142(6):1264.e1261-1273. e1261.

8. Supramaniam R, O'Connell DL, Tracey E, Sitas F. Cancer incidence in New South Wales migrants 1991 to 2001. Sydney: The Cancer Council NSW; 2006.

9. Visser O, van Leeuwen FE. Cancer risk in first generation migrants in North-Holland/Flevoland, The Netherlands, 1995-2004. Eur J Cancer. 2007;43(5):901-908.
10. MacLachlan JH, Allard N, Towell V, Cowie BC. The burden of chronic hepatitis B virus infection in Australia, 2011. Aust NZJ Public Health. 2013;37(5):416-422.

11. Rossi C, Shrier I, Marshall L, et al. Seroprevalence of chronic hepatitis $B$ virus infection and prior immunity in immigrants and refugees: a systematic review and meta-analysis. PLoS One. 2012;7(9):e44611.

12. Kowdley KV, Wang CC, Welch S, Roberts H, Brosgart CL. Prevalence of chronic hepatitis B among foreign-born persons living in the United States by country of origin. Hepatology. 2012;56(2):422-433.

13. European Association for the Study of the Liver. EASL clinical practice guidelines: management of chronic hepatitis B virus infection. $J$ Hepatol. 2012;57(1):167-185.

14. Allard NL, MacLachlan JH, Cowie BC. The cascade of care for Australians living with chronic hepatitis B: measuring access to diagnosis, management and treatment. Aust $N Z J$ Public Health. 2015;39(3):255-259.

15. MacLachlan J, Cowie B. Hepatitis B Mapping Project: Estimates of chronic hepatitis $B$ prevalence and cultural and linguistic diversity by Medicare Local, 2011 - National Report. Sydney: Australasian Society for HIV Medicine; 2013.

16. $\mathrm{Hu} \mathrm{KQ}$, Pan CQ, Goodwin D. Barriers to screening for hepatitis B virus infection in Asian Americans. Dig Dis Sci. 2011;56(11): 3163-3171.

17. IOM (Institute of Medicine). Hepatitis and Liver Cancer: A National Strategy for Prevention and Control of Hepatitis $B$ and $C$. Washington DC: Institute of Medicine; 2010.

18. Robotin MC, Kansil M, Howard K, et al. Antiviral therapy for hepatitis B-related liver cancer prevention is more cost-effective than cancer screening. J Hepatol. 2009;50(5):990-998.

19. MacLachlan J, Cowie B. Hepatitis B Mapping Project: Estimates of Chronic Hepatitis B Diagnosis, Monitoring and Treatment by Medicare Local. National Report 2012/13. Sydney: Australasian Society for HIV Medicine (ASHM) and Victorian Infectious Diseases Reference Laboratory (VIDRL); 2015.

20. Robotin MC, Kansil MQ, George J, et al. Using a population-based approach to prevent hepatocellular cancer in New South Wales, Australia: effects on health services utilization. BMC Health Serv Res. 2010;10:215.

21. Yuen MF, Yuan HJ, Hui CK, et al. A large population study of spontaneous HBeAg seroconversion and acute exacerbation of chronic hepatitis B infection: implications for antiviral therapy. Gut. 2003;52(3):416-419.

22. Robotin M, Patton Y, Kansil M, Penman A, George J. Cost of treating chronic hepatitis B: comparison of current treatment guidelines. World J Gastroenterol. 2012;18(42):6106-6113.

23. Robotin M, Porwal M, Kansil M, Penman A, George J. Communitybased prevention of hepatitis-B-related liver cancer: Australian insights. Bull World Health Organ 2014;92(5):374-379.

24. Robotin M, Patton Y, George J. Getting it right: the impact of a continuing medical education program on hepatitis B knowledge of Australian primary care providers. Int J Gen Med. 2013;6:115-122.

25. Matthews G, Robotin M, editors. B Positive-All You Wanted to Know About Hepatitis B: A Guide for Primary Care Providers. ASHM, ed. Sydney: Australasian Society for HIV Medicine; 2008.

26. Robotin MC, Kansil MQ, Porwal M, Penman AG, George J. Communitybased prevention of hepatitis-B-related liver cancer: Australian insights. Bull World Health Organ. 2014;92(5):374-379.

27. Gastroenterological Society of Australia and Digestive Health Foundation. Australian and New Zealand chronic hepatitis $B(\mathrm{CHB})$ recommendations. Clinical update; 2010.

28. Australian Bureau of Statistics. Fairfield City community profile. profile. id community profile 2015. Accessed 3 July, 2015.

29. Cowie B. The linguistic demography of Australians living with chronic hepatitis B. Aust N Z J Public Health. 2011;35(1):12-15.

30. Robotin MC, Porwal M, Hopwood M, et al. Listening to the consumer voice: developing multilingual cancer information resources for people affected by liver cancer. Health Expect. 2017;20(1):171-182. 
31. Camilloni L, Ferroni E, Cendales BJ, et al; Methods to increase participation Working Group. Methods to increase participation in organized screening programs: a systematic review. BMC Public Health. 2013;13:464.

32. Buster EH, Flink HJ, Cakaloglu Y, et al. Sustained HBeAg and HBsAg loss after long-term follow-up of $\mathrm{HBeAg}$-positive patients treated with peginterferon alpha-2b. Gastroenterology. 2008;135(2):459-467.

33. Gish RG, Cooper SL. Hepatitis B in the Greater San Francisco Bay Area: an integrated program to respond to a diverse local epidemic. J Viral Hepat. 2011;18(4):e40-e51.

34. Bailey MB, Shiau R, Zola J, et al. San Francisco hep B free: a grassroots community coalition to prevent hepatitis B and liver cancer. J Community Health. 2011;36(4):538-551.

35. Chronic Hepatitis B Surveillance Report 2007-2008, San Francisco. San Francisco: Communicable Disease Control \& Prevention Section, San Francisco; September 2009.

36. Chen CJ, Yang HI, Su J, et al. Risk of hepatocellular carcinoma across a biological gradient of serum hepatitis $\mathrm{B}$ virus DNA level. JAMA. 2006;295(1):65-73.
37. Leroy V, Asselah T. Universal hepatitis B vaccination: the only way to eliminate hepatocellular carcinoma? J Hepatol. 2015;63(6):1303-1305.

38. Britt H, Miller G, Henderson J, et al. A Decade of Australian General Practice Activity 2005-06 to 2014-15. Sydney University of Sydney; 2015.

39. Wong VW, Janssen HL. Can we use HCC risk scores to individualize surveillance in chronic hepatitis B infection? J Hepatol. 2015;63(3):722-732.

40. Yang HI, Sherman M, Su J, et al. Nomograms for risk of hepatocellular carcinoma in patients with chronic hepatitis B virus infection. J Clin Oncol. 2010;28(14):2437-2444.

41. Yuen MF, Tanaka Y, Fong DY, et al. Independent risk factors and predictive score for the development of hepatocellular carcinoma in chronic hepatitis B. J Hepatol. 2009;50(1):80-88.

42. Yang HI, Lee MH, Liu J, Chen CJ. Risk calculators for hepatocellular carcinoma in patients affected with chronic hepatitis B in Asia. World J Gastroenterol. 2014;20(20):6244-6251.

\section{Clinical Epidemiology}

\section{Publish your work in this journal}

Clinical Epidemiology is an international, peer-reviewed, open access, online journal focusing on disease and drug epidemiology, identification of risk factors and screening procedures to develop optimal preventative initiatives and programs. Specific topics include: diagnosis, prognosis, treatment, screening, prevention, risk factor modification,

Submit your manuscript here: https://www.dovepress.com/clinical-epidemiology-journal

\section{Dovepress}

systematic reviews, risk and safety of medical interventions, epidemiology and biostatistical methods, and evaluation of guidelines, translational medicine, health policies and economic evaluations. The manuscript management system is completely online and includes a very quick and fair peer-review system, which is all easy to use. 